\title{
Influence of self-organizing microstructures on the wettability of molten plastic on steel for hybrid plastic-metal joints
}

\author{
K. van der Straeten ${ }^{1}$ (D) J. Sparla ${ }^{1} \cdot$ A. Olowinsky ${ }^{1} \cdot$ A. Gillner ${ }^{1}$ \\ Received: 15 October 2018 / Accepted: 5 June 2019 / Published online: 15 June 2019 \\ (C) The Author(s) 2019
}

\begin{abstract}
Multi-material construction enables new approaches to lightweight production by using the right material in the right spot. In order to combine different materials suitable joining techniques are required, e.g., for plastics and metals. The production of plastic-metal hybrid enables reducing dead weight while maintaining and preferably boosting the components performance. Common joining techniques like adhesive joining and riveting have specific disadvantages and direct welding of these materials fails due to their different physical and chemical properties. Another promising approach is a two-staged laser-based joining process. First, a laser source generates microstructures on the metallic joining partner to increase the boundary surface and create undercut cavities. In the second step plastic and metal are thermally joined together. Both joining partners are clamped together, the metal surface is heated up with a laser, and through heat conduction, the thermoplastic polymer matrix melts and flows into the cavities. After hardening, a connection is formed. For metal surface microstructuring, ultrashort pulsed lasers can be used to create a spongy topography. These self-organizing microstructures, so-called cone-like protrusions, have previously shown to have a big influence on the wettability of the surface. For hybrid joining, it is essential to have a complete filling of the microstructures with molten polymer in order to achieve high joint strengths, which can be achieved by a good wettability of the metal surface. In this contribution, the wettability of molten plastic (PA6 and PP) on microstructured steel surfaces is evaluated to identify the correlation between wettability and joint strength.
\end{abstract}

Keywords Hybrid joining $\cdot$ Plastic-metal joining $\cdot$ Laser microstructuring $\cdot$ Wettability $\cdot$ Laser joining

\section{Introduction}

The reduction of weight through innovative lightweight construction concepts is an important topic, especially for automotive and aeronautical industry. One way to reduce weight is by using the right material in the right place. The combination of different materials, such as plastics and metals, adapted to local loads allows new approaches for weight optimization [1]. Whereas on the one hand, polymers are characterized by low weight, attractive price, and nearly endless shaping possibilities,

Recommended for publication by Commission XVI - Polymer Joining and Adhesive Technology

K. van der Straeten

kira.van.der.straeten@ilt.fraunhofer.de

1 Fraunhofer Institute for Laser Technology ILT, Steinbachstr. 15, 52074 Aachen, Germany metals on the other hand can withstand significantly higher loads due to their very good mechanical properties [1-3]. However, a direct and firm joint between both materials fails due to their physical and chemical diversity. Nowadays, joining material combinations of fiberreinforced polymers (FRP) and metals (steel, aluminum) is mostly based on mechanical fastening or adhesive joining [1-3]. Since mechanical fasteners have a punctual load transfer, destroy fibers, and weaken the material's mechanical properties, they are not suitable for the use of fiber-reinforced plastics. On the other hand, adhesive joints need extensive surface pretreatments and curing times. In order to realize the multi-material lightweight approach, suitable joining techniques are required. A promising approach to overcome these problems is a laser-based two-step process consisting of metal surface microstructuring and direct thermal joining [1-3]. For this method, high joint strengths between different materials of approx. $25 \mathrm{MPa}$ have already been proven in previous works $[1,2]$. This process can be used for joining plastics 
and metals for different applications like structural parts like doors, car body parts, and seating structures, or small parts like valves, housings, and bearings. As microstructures generated by the laser microstructuring process influence the wetting properties of the metal surface, this might have an influence on the strength of the hybrid joint. In this contribution, the influence of the microstructures on the wettability of the steel surface is investigated.

\section{Fundamentals}

\subsection{Laser-based plastic-metal joining}

The laser-based process chain is shown in Fig. 1a. Within the first process step the metal surface is microstructured with laser radiation to create undercut structures for the interlocking of polymer and metal and to enlarge the boundary surface and create undercut structures [1-3]. In a second heat conduction joining process step, the metal is heated up and by thermal contact of polymer and metal the thermoplastic composite material melts [1-3]. Applying an external clamping force, the plasticized material flows into the structures. After resolidification, a direct and firm bond is formed [1-3].

For microstructuring of the metal surface, different approaches are investigated which differ in used laser source and processing strategy. In this contribution, ultrashort laser pulses are used to create a spongy surface consisting of microstructures, partially covered with nano-substructures (see Fig. 1b) like already used in previous works [1-3]. These socalled cone-like protrusions (CLP) appear during ablation of metals or silicon with ultrashort pulsed laser sources at medium $\left(\sim 1 \mathrm{~J} / \mathrm{cm}^{2}\right)$ and high fluences $[4,5]$. Usually, the effect starts at grooves, scratches, and inhomogeneities after a few layers of ablation with high-energy ultrashort pulses in the ablated area. At first, some dots and holes appear which grow to small clusters with an increasing number of layers until they completely cover the surface. Usually, the depth of these microstructures varies between 50 and $150 \mu \mathrm{m}$ and the width between 10 and $50 \mu \mathrm{m}$ [2]. Visually, the growth of the CLP on steel can be monitored due to a blackening of the surface. The structure is characterized by a random orientation and an extremely enlarged surface with a high surface roughness, which enables points for mechanical interlocking with plastics for hybrid joints [1-3].

\subsection{Wetting of rough surfaces}

Wetting is the behavior of liquids at the boundary of a solid. The wettability of solid surfaces with a liquid phase can be described by the Young contact angle $(\Theta)$. Figure 2 shows the respective contact angles of different wetting behavior [6].

The Young contact angle is defined for an ideal, perfect smooth, and chemically homogeneous surface. Real surfaces, especially microstructured surfaces, do not have these ideal properties. The wetting behavior of rough surfaces is described by Wenzel and Cassie-Baxter [7, 8]. Wenzel assumes a homogeneous wetting of the rough surface structure and thus describes the complete penetration of the liquid into the structure [7]. However, sometimes, air is trapped between the solid and the liquid, especially on hydrophobic surfaces. The liquid does not penetrate into the spaces between the structures and thus does not wet the entire surface. This type of wetting is called heterogeneous wetting and is described by Cassie-Baxter [8].

Figure 3 illustrates the homogeneous wetting as Wenzel stage and the heterogeneous wetting as the Cassie-Baxter stage. Due to an additional energy effect, a heterogeneous wetting according to Cassie-Baxter can change into a homogeneous wetting according to Wenzel.

\section{Experimental setup and procedure}

\subsection{Materials and sample geometry}

In this contribution, the metal component for all trials is a commercially available stainless steel (X5CrNi18-10, type

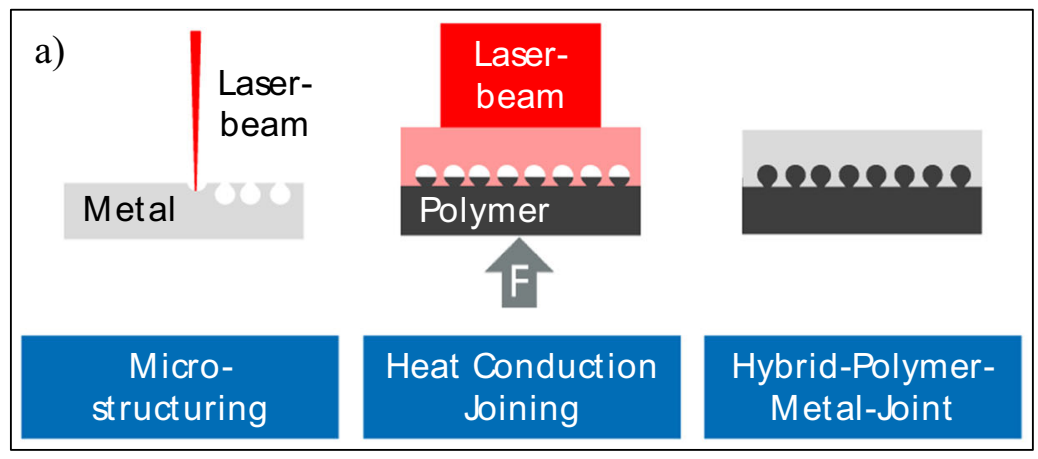

b)

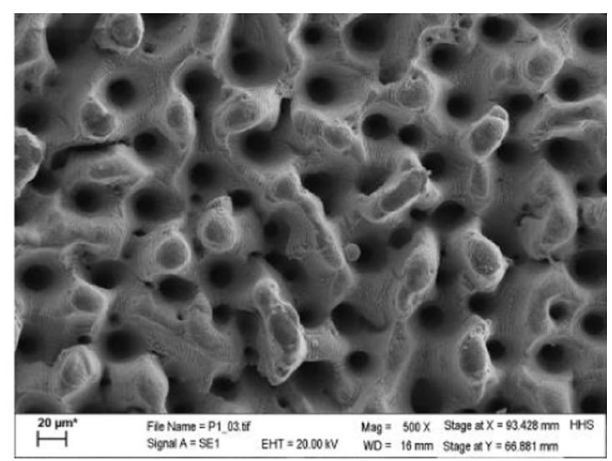

Fig. 1 a Process chain of the laser-based plastic-metal connection. b Cone-like protrusions (CLP) on stainless steel (SEM-picture) 

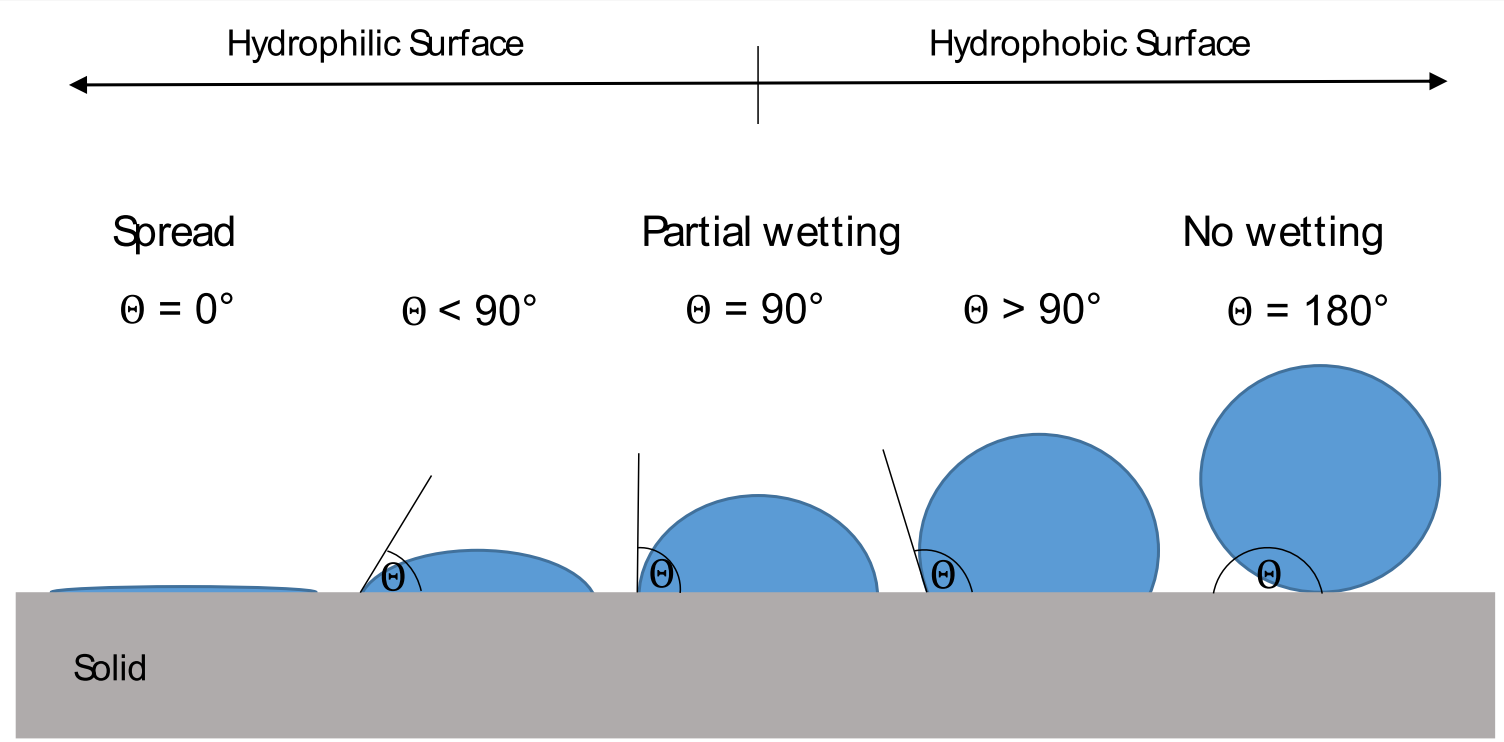

Fig. 2 Contact angles of different wetting behaviors [6]

1.4301 ) with a material thickness of $1.5 \mathrm{~mm}$. For wetting tests, squared areas of $5 \times 5 \mathrm{~mm}^{2}$ are microstructured using ultrashort pulsed lasers as described in 3.2 (see Fig. 4a), where drops can be placed and the wetting properties can be analyzed. Tensile shear tests are used to investigate the influence of the wetting properties on the joint strength. Therefore, a joining area of $30 \times 5 \mathrm{~mm}^{2}$ is microstructured in the overlapping area of the sample (see Fig. 4b).

The wetting analysis is carried out with distilled water and with thermoplastics polyamide 6 (PA6) and polypropylene (PP). The plastics are provided as unreinforced plastic granules:

- PA6 Ultramid B3S (BASF), cylindrical granules, length 3.0-3.2 mm, diameter 2.0-2.1 mm, melting temperature $220{ }^{\circ} \mathrm{C}$

- $\quad$ PP HD120MO (BOREALIS), lenticular granules, length 4.0-4.3 $\mathrm{mm}$, diameter 3.3-3.6 mm, melting temperature $230-260{ }^{\circ} \mathrm{C}$

For tensile shear tests, glass fiber-reinforced plastics are used:

Fig. 3 Wetting stages according to Wenzel and Cassie-Baxter
- PA6 Tepex dynalite 102-RG600(x) (bond laminates) with a glass fiber volume content of $47 \%$ and 2-mm material thickness (PA6/GF47). The glass fibers are arranged as woven fabric with $0 / 90$ orientation.

- $\quad P P$ X111F40-4/1-0/90 (quadrant plastic composites AG), which consists a chopped fiber glass mat-reinforced PP laminate with randomly oriented glass fibers and additionally reinforced fabric inside. The material thickness is $3 \mathrm{~mm}$ with a glass fiber content of 40 vol.\% (PP/GF40).

To achieve a hybrid joint sample for tensile shear testing purposes, plastic and metal are arranged in an overlap configuration (Fig. 4b).

\subsection{Experimental setup for microstructuring}

Microstructuring of the sample surfaces is carried out with two different ultrashort pulsed beam sources. The first system used consists of a Kugler Microgantry nano3X (3-axis motion system) with an ultrashort pulsed laser Hyper Rapid 100 (Coherent). Pulse duration is $<15$ ps at a wavelength of $\lambda=$ $1064 \mathrm{~nm}$. The maximum average output power is $100 \mathrm{~W}$ at a
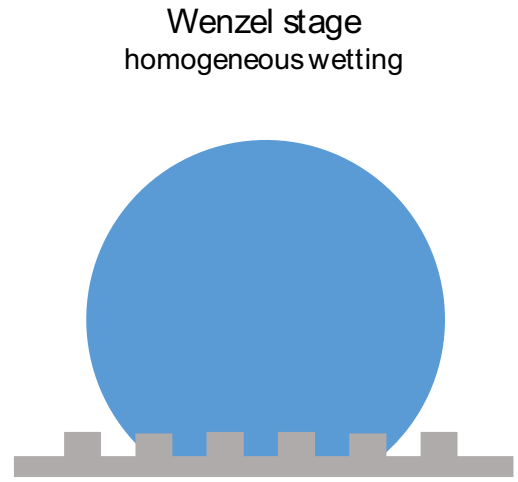

Cassie-Baxter stage heterogeneous wetting

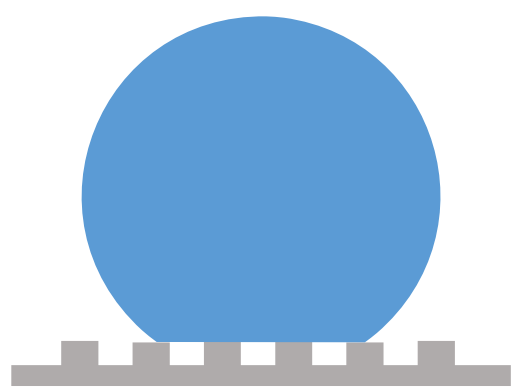



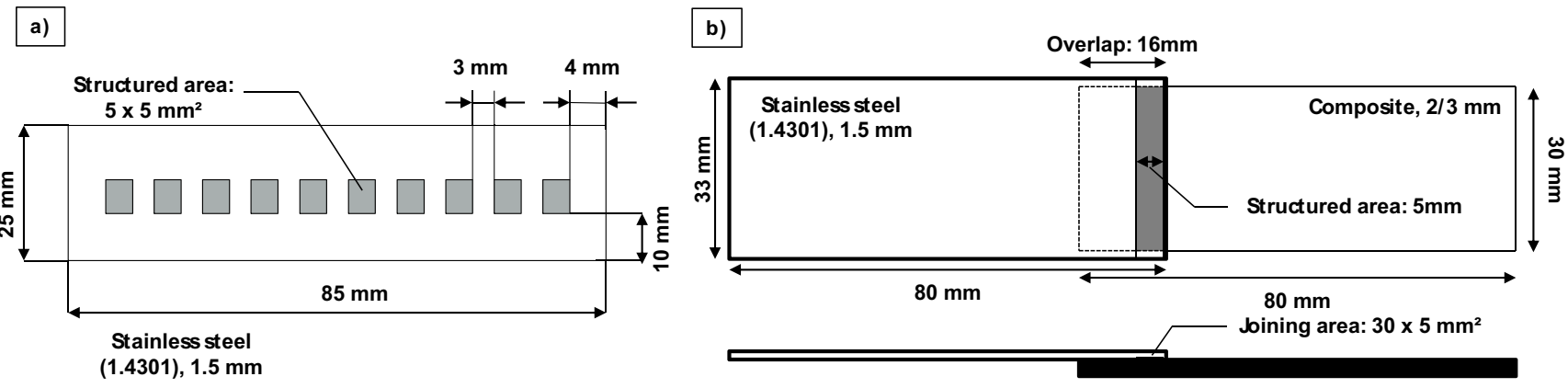

Fig. 4 a Sample geometry for wetting tests. b Sample geometry for tensile shear tests

repetition rate of $400 \mathrm{kHz}$. After deflection by a galvanometric scanner (IntelliScan14, Scanlab), the laser beam is focused through a lens $(f=163 \mathrm{~mm})$ to a spot size of approx. $34 \mu \mathrm{m}$.

The second system consists of a DMG Sauer Lasertec50 (5-axis motion system) with an ultrashort pulsed laser Trumicro 5050 (Trumpf Lasertechnik GmbH). The pulse duration is about $6 \mathrm{ps}$ at a wavelength of $\lambda=1030 \mathrm{~nm}$. The maximum average power is $50 \mathrm{~W}$ at a maximum repetition rate of $800 \mathrm{kHz}$. After deflection by a galvanometric scanner (IntelliScan14, Scanlab), the laser beam is focused through a lens $(f=100 \mathrm{~mm})$ on the work piece to a spot size of approx. $25 \mu \mathrm{m}$.

Variable parameters to optimize the process window for the formation of CLP are scanning speed $v$, structure offset SO, laser power $P$, and the number of ablated layers $N$. The samples are processed with a crossed scanning hatch. The choice of microstructuring parameters are based on preliminary tests and the number of ablated layers $N$ is varied. The parameters used are listed in Table 1. After the microstructuring process, the surface roughness (mean surface roughness Ra) is evaluated using a laser scanning microscope (LSM). The resulting 3D profile images do not completely reflect the depth profile due to the undercuts and deep cavities, but can be used for approximate evaluation of the roughness profile.

\subsection{Experimental setup for wetting analysis}

The wetting analysis of a microstructured surface is first carried out with distilled water. The advantage is that the properties of distilled water are constant and wetting can take place at room temperature, thus simplifying handling and the results can be compared with those of wetting with molten plastic.
To perform the wetting analysis, a contact angle measuring instrument OCA20 (DataPhysics Instruments $\mathrm{GmbH}$ ) is used (see Fig. 5). The oven for heating the sample table is removable. The contact angle measuring instrument has an electronically controllable dosing unit containing the syringe cartridge from Hamilton, model DS500/GT, with a volume of $500 \mu \mathrm{l}$. For distilled water measurements, the syringe cartridge is fitted with a Nordson SNS-D 051/025 cannula with an internal diameter of $0.51 \mathrm{~mm}$ and an external diameter of $0.525 \mathrm{~mm}$. Using the software SCA20 from DataPhysics Instruments $\mathrm{GmbH}$, a defined quantity of $2 \mu \mathrm{l}$ of water is deposited on the sample placed on the sample table. The contour of the drop is captured by the horizontally aligned camera by means of a diffuse light source mounted at the opposite of the camera lens. The evaluation of the drop shape and the contact angle is also carried out with the software SCA20. The sample stage is aligned by manual vertical and horizontal sample stage adjustment in such a way that the drop to be detected is detected centrally by the camera.

For measurements with molten plastic, due to its high viscosity, the plastic melt does not constrict at the tip of a ceramic cannula and thus does not form a drop but is pressed out in the form of a strand. Instead, the plastic granulate is deposited on the structured sample surface and heated to a defined melting temperature in the preheated oven. After a fixed heating time (see Table 2), the plastic granulate melts completely and forms a drop. The sample is then removed from the oven and cooled at room temperature. For evaluation, the sample is analyzed without an oven. The cannula for the measurements with water serves as a reference value for the optical evaluation.

The Laplace-Young fitting evaluation method is used in the SCA20 software for a uniformly rounded drop (water, PA6). This evaluation method automatically detects the drop contour
Table 1 Laser microstructuring parameters

\begin{tabular}{lllll}
\hline Laser source & Av. laser power $(\mathrm{W})$ & $\begin{array}{l}\text { Scanning speed } \\
(\mathrm{mm} / \mathrm{s})\end{array}$ & Structure offset $(\mu \mathrm{m})$ & Ablated layers (\#) \\
\hline Hyper Rapid 100 & 57 & 2000 & 8 & $2-30$ \\
Trumicro 5050 & 47.5 & 2000 & 8 & $2-30$ \\
\hline
\end{tabular}


Fig. 5 System setup for contact angle measurement

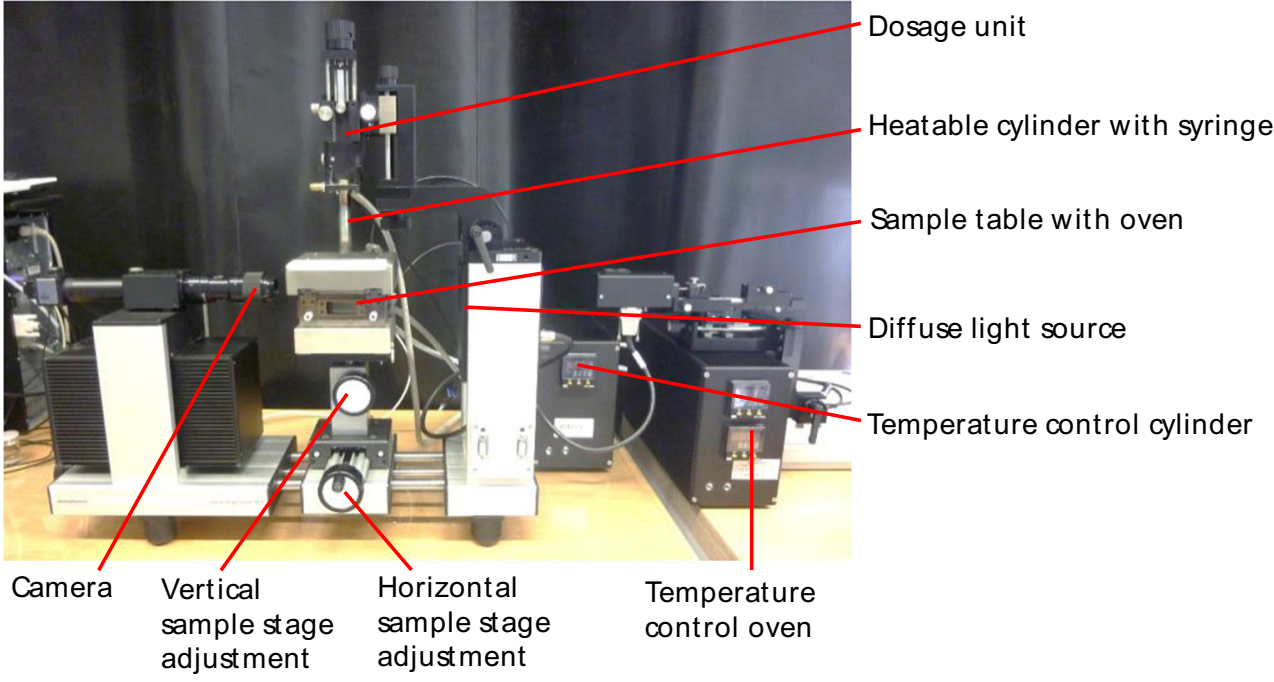

and the baseline between drop and wetting surface. The contact angle is then determined by the software. The dimensions of the cannula used are stored in the software and serve as a reference value. In contrast to water and PA6 drops, PP forms an unevenly rounded drop shape. The PP drop shape is elliptical and can be evaluated either by contact angle computation (CA computation) or by a manual evaluation method.

\subsection{Experimental setup for joining}

In order to establish a connection between the determined wetting properties and the attainable joining strength of hybrid joints, tensile shear specimens are made of glass fiberreinforced PA6/PP and stainless steel. The plastics are used as fiber-reinforced variants to ensure the necessary strength during the tensile shear test. The laser system used for the joining process is a LDM 3000-100 (Laserline $\mathrm{GmbH}$ ) continuous wave diode laser combined with a MOTOMANHP20-6 robot motion system. The maximum output power is $3000 \mathrm{~W}$ and the laser system is operating at $\lambda=900$ $1070 \mathrm{~nm}$ wavelength. A zoom optics (Laserline $\mathrm{GmbH}$ ) with a focal length of $250 \mathrm{~mm}$ forms a rectangular laser spot with a variable size from $5 \times 5 \mathrm{~mm}^{2}$ up to $16 \times 30 \mathrm{~mm}^{2}$. The variable spot size enables simultaneous irradiation of the entire joining area with a single pulse adapted to the sample geometry $(30 \times$ $5=150 \mathrm{~mm}^{2}$ ). In order to apply pressure and fix the sample arrangement, a pneumatic clamping device with a sample holding fixture is used with a clamping pressure of 3 bars on

Table 2 Parameters for plastic droplet formation

\begin{tabular}{lll}
\hline Polymer & PA6 & PP \\
\hline Oven temperature $\left({ }^{\circ} \mathrm{C}\right)$ & 235 & 250 \\
Heating time (s) & 180 & 120 \\
\hline
\end{tabular}

the joining area. The parameters chosen for joining are based on the preliminary tests and are listed in Table 3 . There is no cooling applied after joining.

The mechanical properties (tensile shear strength) of the hybrid joints are tested with a multi-purpose testing machine Z100 (Zwick GmbH \& Co. KG). For the shear strength tests, a tensile force is applied parallel to the joining area and the breaking force is measured according to DIN EN 1465. The samples are fixed between the clamping jaws at a $50-\mathrm{mm}$ distance and testing speed is set to $50 \mathrm{~mm} / \mathrm{min}$. Five samples are tested for each parameter-material combination.

\section{Results and discussion}

\subsection{Formation of CLP on steel}

The formation of CLP on steel is a growth process. With an increasing number of ablated layers $(N)$, the dots appear on the metal surface which grow to a spongy microstructure. The formation of CLP on steel using the Hyper Rapid 100 is shown in Fig. 6. While for $N=10$ passes, only few dots are visible, and with $N=12$ passes, a lot of structures appear on the metal surface which grow to a completely covered surface at $N=14$. After that point, the structure only grows deeper, the cone-like dots get higher, and the bridges

Table 3 Laser joining parameters

\begin{tabular}{lll}
\hline Material & Laser power* (W) & Irradiation time (s) \\
\hline PA6/GF47 & 2550 & 2 \\
PP/GF40 & 2300 & 2 \\
\hline
\end{tabular}

*Laser power at work piece (after optics) 


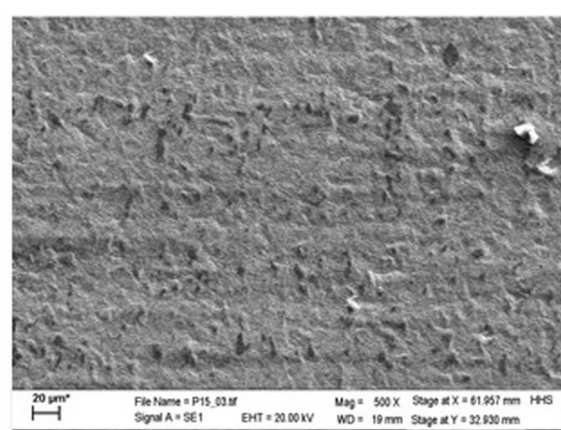

$N=2$

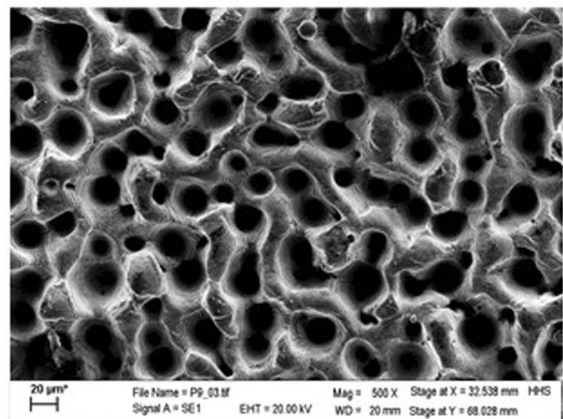

$N=14$

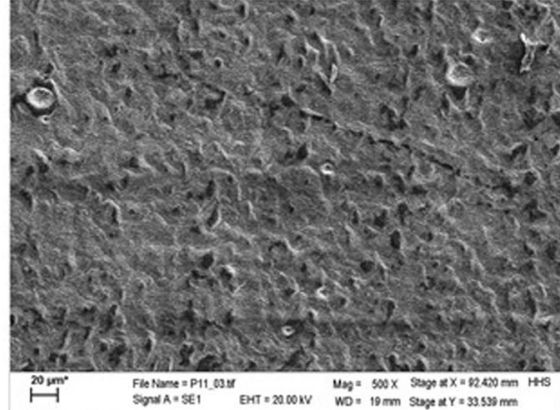

$N=10$

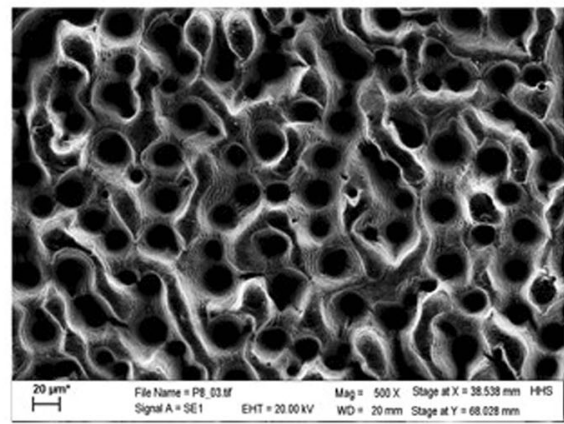

$N=16$

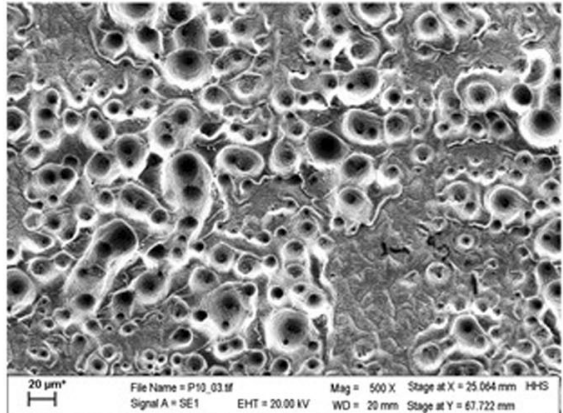

$N=12$

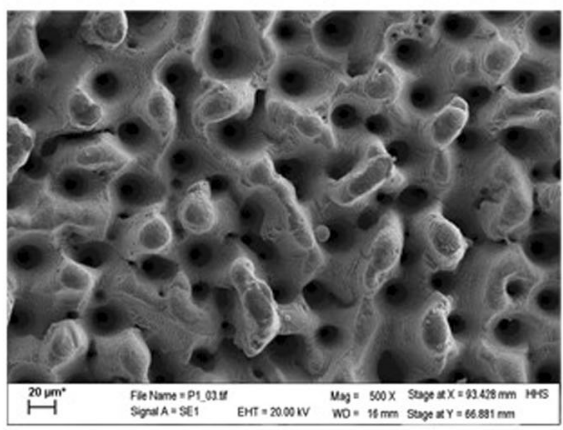

$N=30$

Fig. 6 SEM pictures of the formation of CLP on steel with an increasing number of ablated layers (Hyper Rapid 100)

between the dots become lower. The microstructures themselves are covered with nano-substructures and have overall smooth, round curves.

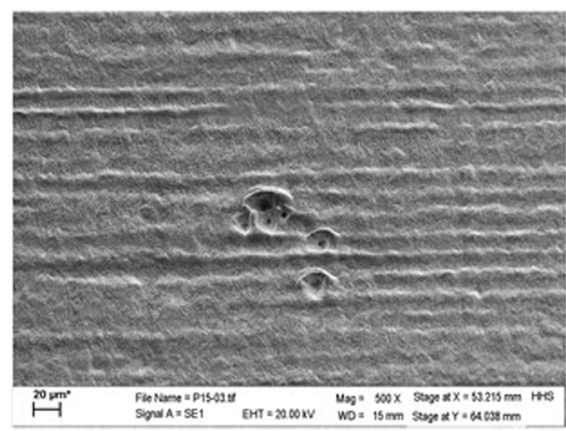

$$
N=2
$$

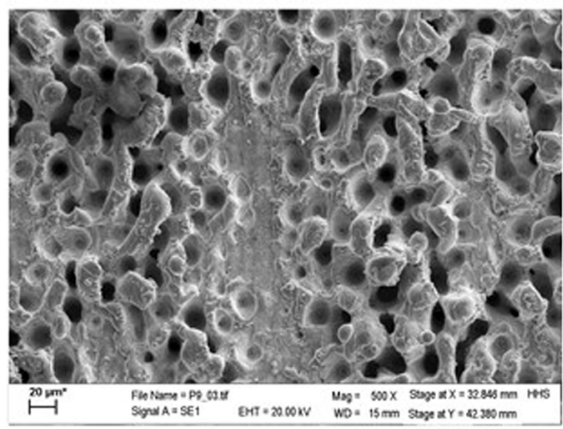

$$
N=14
$$

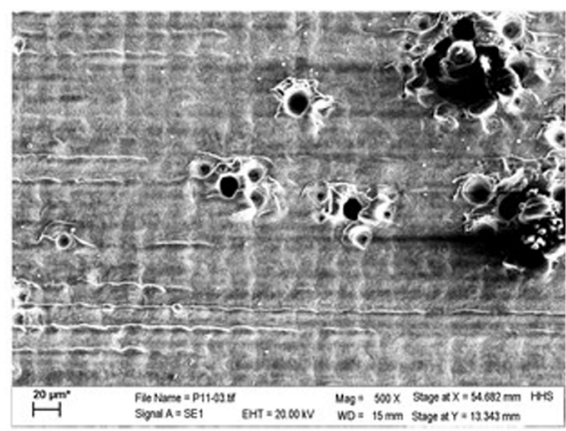

$$
N=10
$$

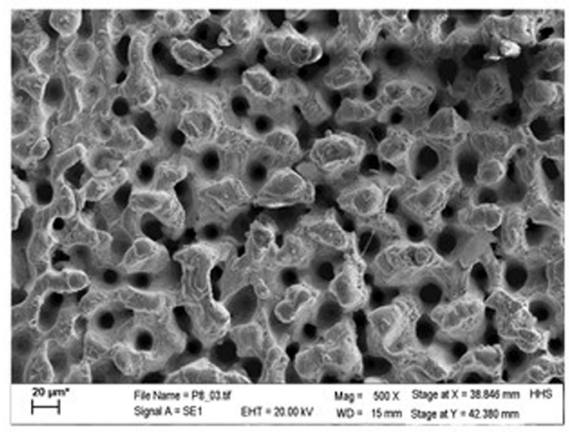

$N=16$
The formation of CLP on steel using a Trumicro5050 is similar to using a Hyper Rapid 100 (see Fig. 7). After a few passes $(N=2)$, first defects and dots appear on the surface.

Fig. 7 SEM pictures of the formation of CLP on steel with an increasing number of ablated layers (Trumicro 5050) 
Between $N=10$ and $N=16$ layers, the structures grow rapidly until they completely cover the metal surface. The microstructures are also covered with nano-substructures, but are not as smooth as those made with the Hyper Rapid 100.

The CLP structures are characterized by a random orientation and an extremely enlarged surface. Without magnification, the surface appears completely black due to the high light absorption. In Fig. 8, cross-sections of the microstructured surfaces are shown for both laser sources used at $N=28$ ablated layers. The holes of the microstructures are between $20 \mu \mathrm{m}$ and $160 \mu \mathrm{m}$ deep and the width varies between a few microns up to $30 \mu \mathrm{m}$.

Comparing the cross-sections of both beam sources, the results are comparable with the SEM pictures: the Hyper Rapid 100 structures are more even and look like cones and the Trumicro 5050 look similar but are more rough and uneven.

\subsection{Results of wetting analysis}

\subsubsection{Wetting with distilled water}

The microstructured surface is analyzed via laser scanning microscopy. The roughness profile of the microstructuring variations makes a decisive contribution to understanding the wetting analysis results. Figure 9 a shows the evaluation of the mean roughness value of the individual microstructured surfaces. The graph marked in blue corresponds to the mean roughness value of the Hyper Rapid 100 structures, and the green graph to the mean roughness value of the Trumicro 5050 structures. The course of the mean roughness value is similar for the fabricated structures of both laser sources and corresponds to the course of CLP formation previously observed in the SEM image evaluation. The mean roughness value represents the volatile rise during CLP development process.

The evaluation of the wetting analysis with water is shown in Fig. 9b. The graph marked in blue again corresponds to the contact angle of the Hyper Rapid 100 structures, and the green

a)

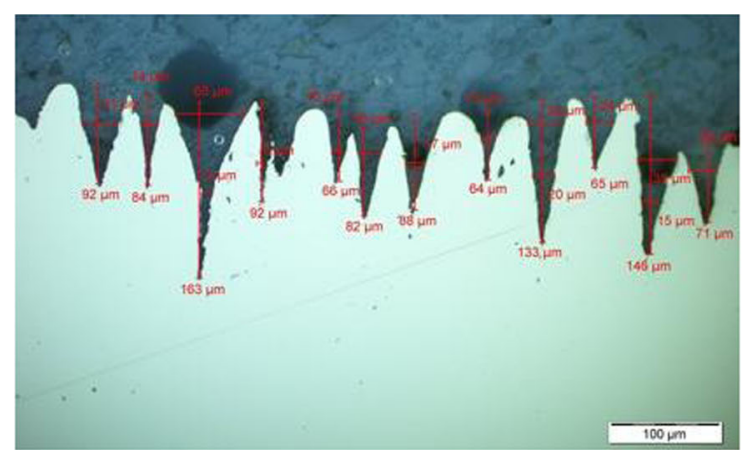

graph to the contact angle value of the Trumicro 5050 structures. Each measuring point represents the average of 10 individual measurements. The course of the determined contact angles corresponds to the course of the average roughness values of the respective surface textures shown in Fig. 9a. A significant increase in the contact angle can be seen as soon as the surface is completely covered with CLP structures. If the CLP structures are not yet fully grown, contact angles in a range of $80-90^{\circ}$ are measured. With a completely covered CLP structure, contact angles are measured in a range of $130-140^{\circ}$. Both microstructure types have contact angles at a comparable level. The CLP structures thus cause the contact angle to increase by approx. $50^{\circ}$. The already existing hydrophobicity is clearly increased by the microstructuring process.

\subsubsection{Wetting with PA6}

The evaluation of the wetting analysis with PA6 is shown in Fig. 10a. When wetting with PA6 plastic melt, the characteristic course of the contact angle corresponds to the course of wetting with water. As soon as the CLP structures have full surface coverage, the determined contact angles increase. Compared with wetting analysis with water, the increase in the contact angle of the Hyper Rapid 100 structures is somewhat flatter. The measured contact angles of the these structures lie in a range of $120-130^{\circ}$ before the rise, and those of the Trumicro 5050 structures at approx. $110^{\circ}$. If the surface is completely covered with CLP, the contact angles of the Hyper Rapid 100 structures are in a range of $150-160^{\circ}$, and those of the Trumicro 5050 structures in a range of $140-150^{\circ}$. The CLP structures therefore cause an increase in the contact angle of approx. 30-40 . The increase of the standard deviation compared with the wetting with water might be caused by size variations of the granulate/drop, which is less reproducible than the water drops. The standard deviation also rises for $N=14-16$, because this is the transition area where the microstructures grow from small isles to completely covering the surface. The deviation is increased due to the variation of the surface structures in this process regime.

b)

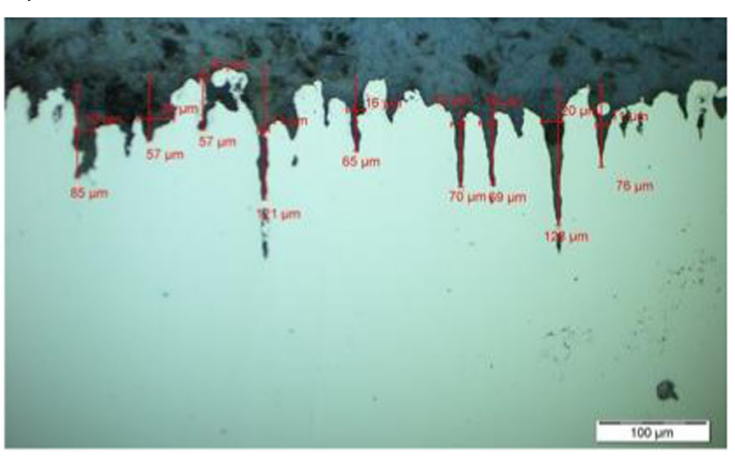

Fig. 8 Cross-sections of the microstructured area for $N=28$ ablated layers with a Hyper Rapid 100 and $\mathbf{b}$ Trumicro 5050 
a)

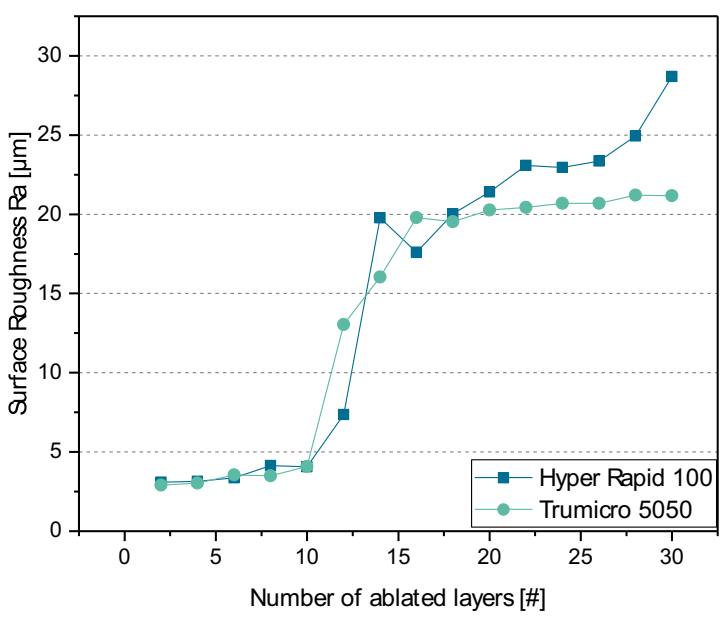

b)

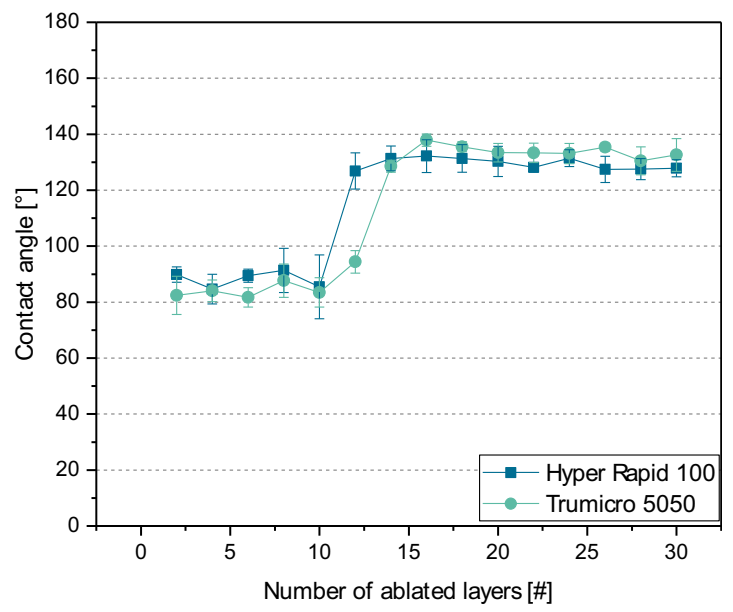

Fig. 9 The mean surface roughness Ra of the metal surface (a). Wetting with distilled water (b)

To enable dividing the wetted samples into homogeneous and heterogeneous wetting (see Fig. 3), cross-sections of the wetted samples are prepared. Figure $10 \mathrm{~b}$ depicts a crosssection of a PA6 drop on the microstructured steel surface (Hyper Rapid 100, $N=26$ ). The drop lies on top of the structures and the plastic does not penetrate into the cavities. This is heterogeneous wetting according to Cassie-Baxter. The microstructured surface by using the Trumicro 5050 shows the same result.

\subsubsection{Wetting with PP}

The evaluation of the wetting analysis with PP is shown in Fig. 11a. The wetting analysis with PP does not show the characteristic course of the previously performed wetting analysis with water and PA6. The results of the wetting analysis with PP are not clearly dependent on the CLP structures. During drop formation, two different drop types/shapes are produced (see Fig. 11b). Due to the different drop shapes, the contact angles are not uniform. The applied PP granules form irregularly shaped drops which can form contact angles of 40 to $150^{\circ}$ on the structured surface. A regularity of this effect is not discernible. The different drop types might be caused by the bigger size of the PP granules so that the surface tension is not sufficient to form a reproducible drop.

Figure 12 depicts cross-section of PP drops on the microstructured surface at different states of the CLP formation (number of ablated layers $N=12,14,26$ ). The crosssections show again the different drop types of PP. In Fig. 12 a, drop type 1 is visible and in Fig. 12 b and c, drop type 2 is present. In all three cross-sections, the cavities of the structures are completely filled with plastic, regardless of the characteristics of the CLP structures. For the combination of $\mathrm{PP}$ with microstructured steel, it is a homogeneous wetting according to Wenzel. a)

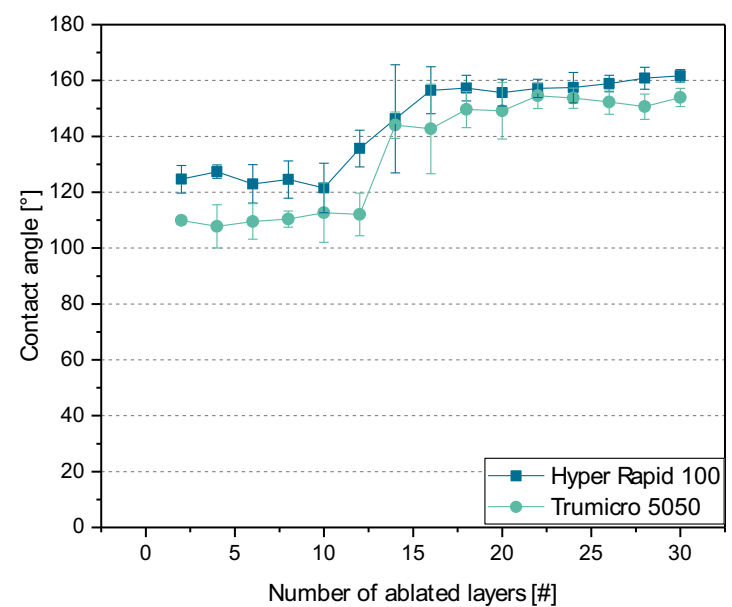

b)

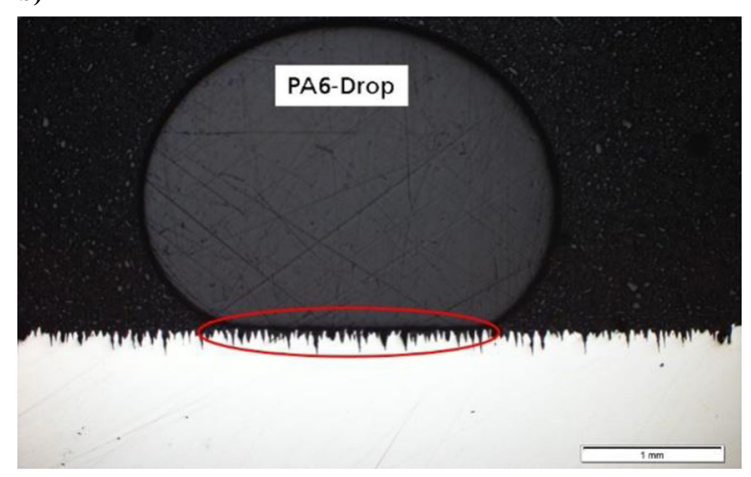

Fig. 10 a Contact angles of wetting with PA6 with an increasing number of ablated layers. b Cross-section of PA6 drop on microstructured surface (Hyper Rapid 100, $N=26$ ) 
a)

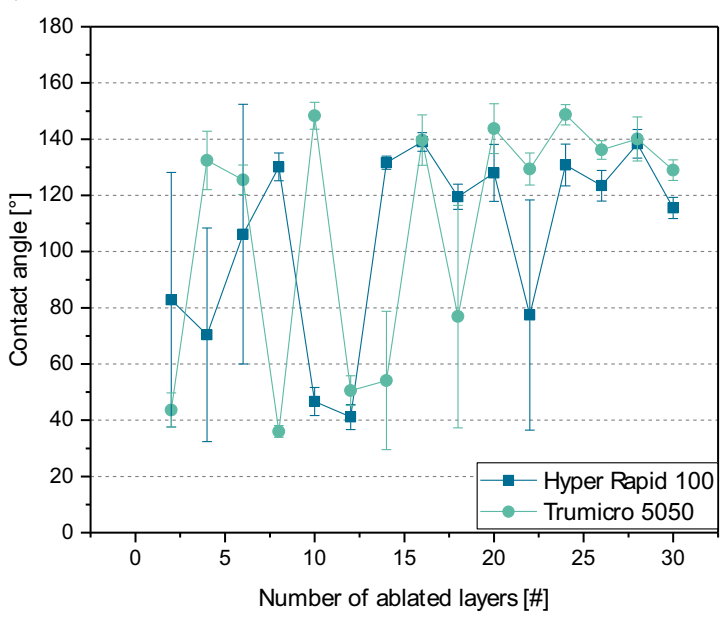

b)

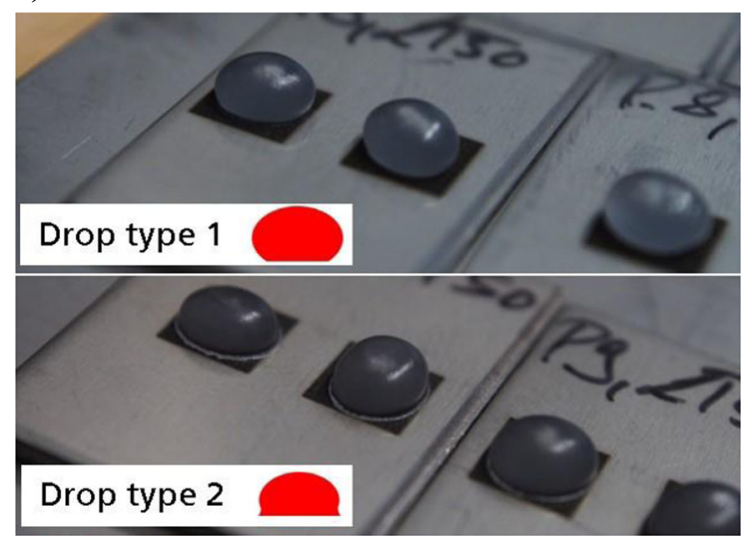

Fig. 11 a Contact angles of wetting with PP with an increasing number of ablated layers. b Different drop types/shapes for PP

\subsection{Results for tensile shear tests}

The results for the tensile shear tests of the samples are depicted in Fig. 13. Figure 13 a shows the joint strength of the hybrid joints between steel and PA6/GF47. The joint strength varies widely and has a large standard deviation independent of the laser source used. With an increasing number of ablated layers, the strength of the joint decreases. These results are contradictory to previous works [2], where joint strengths above $25 \mathrm{MPa}$ between similar materials were achieved. The cause for this can be unsuitable joining parameters which lead to an insufficient filling of the microstructures or aged or wet materials. Additionally, the arrangement of the fibers in the contact area of the joint may influence the joint strength. In comparison with the contact angle measurements, it could be concluded that with increasing contact angle the joint strength decreases. But as the results do not comply with previous results and show a strong deviation, the resilience of the results is not fully given. Further investigations are necessary to determine the cause of these results.

In Fig. $13 \mathrm{~b}$, the tensile shear strength of hybrid joints between PP/GF40 and steel is shown. Overall joint strengths between 22 and $25 \mathrm{MPa}$ are achievable. The standard deviation is much smaller compared with the PA6 samples. The joint strength is comparable for both laser sources used. Contradictory to previous works [2], the complete coverage of the surface with CLP structures is not necessary to achieve high joint strengths.

Summing up, for hybrid joints, it can be concluded that there is no direct influence of the metal's wetting properties of the metal surface on the joint strength, although the surfaces show a strong hydrophobic behavior. For a good joint strength, a filling of the microstructures and therefor a homogeneous wetting should be desired. By applying an additional energy effect, a heterogeneous wetting can be transformed into a homogeneous wetting. In this case, the applied joining pressure serves as additional energy effect and ensures the wetting and filling of the microstructures.

\section{Summary and outlook}

Laser-based joining of plastics and metals enables connecting both materials without any additional materials a)

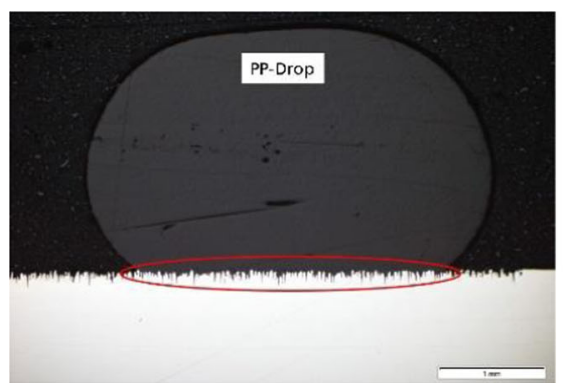

b)

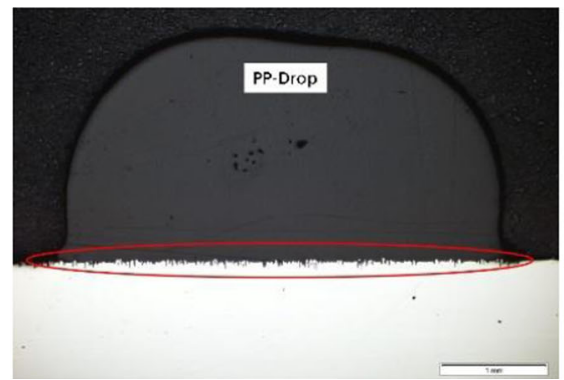

c)

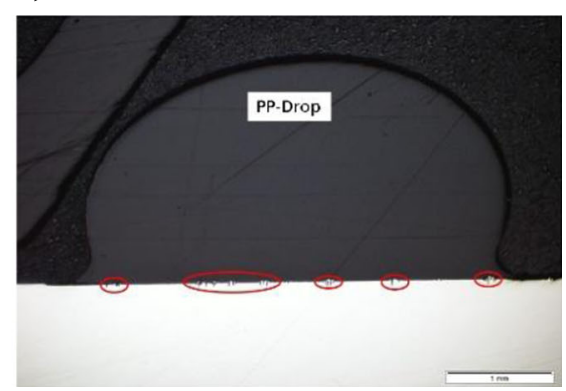

Fig. 12 Cross-section of PP drop on microstructured surface of a Trumicro 5050, $N=26$, $\mathbf{b}$ Trumicro 5050, $N=14$, and $\mathbf{c}$ Trumicro 5050, $N=12$ 
a)

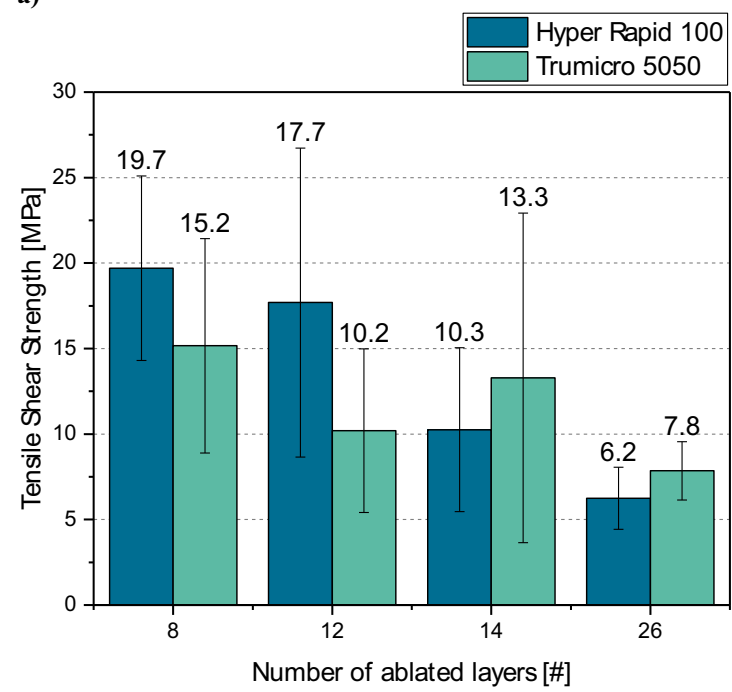

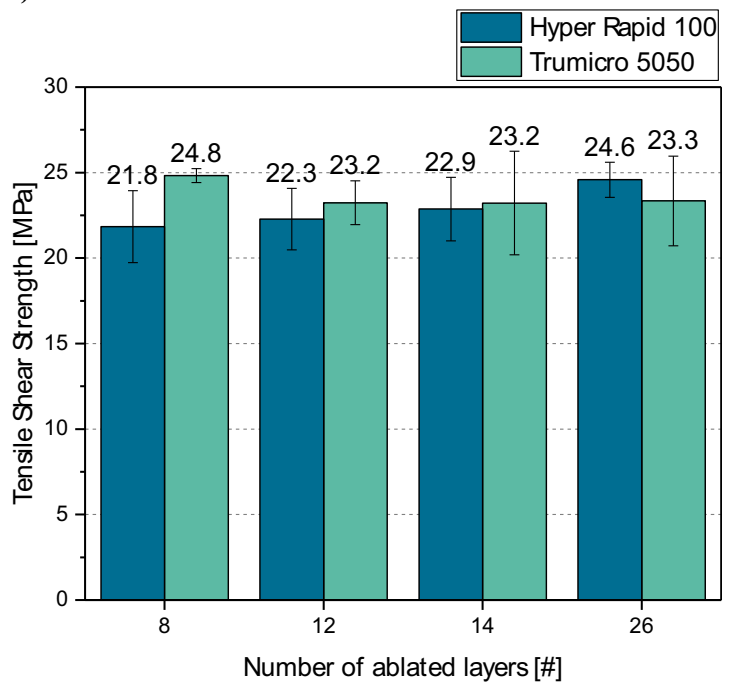

Fig. 13 a Tensile shear strength of hybrid joint with PA6/GF47 and steel. b Tensile shear strength of hybrid joint with PP/GF40 and steel

such as adhesives or fasteners in a two-staged process. First, the surface is microstructured with ultrashort laser pulses to create self-organizing microstructures which provide interlocking points and an enlarged surface. In the subsequent joining process, both materials are clamped together and heated up until the polymer melts and flows into the structures. As microstructures do influence the wettability of surfaces, in this contribution, the influence of selforganizing microstructures on the wettability of molten plastic on steel is investigated.

Two different ultrashort pulsed laser sources are used to generate Cone-like protrusions (CLP) on stainless steel (1.4301). The wetting behavior of the surface during microstructure formation is analyzed using a contact angle measuring instrument. The surfaces produced are wetted with distilled water, PA6 and PP, and the contact angle is measured and correlated with surface roughness. For both laser sources used, the formation of the microstructures rises sharply between 10 and 16 number of ablated layers, which also leads to a strong increase in surface roughness. This rise is also visible during wetting with distilled water and PA6, where the contact angle increases about $50^{\circ}$ up to $140^{\circ}$ for water and $30-40^{\circ}$ up to approx. $150^{\circ}$ for PA6. Contact angles $>90^{\circ}$ are referred to as hydrophobic. For wetting with PP, no clear trend is visible, because two different types of drop shapes form which leads to a strong variation in the measurement of the contact angle. These results might be caused by the too big size of the drops. Cross-sections of the wetted surface with PA6 and PP drops show a heterogeneous wetting according to CassieBaxter for PA6 and a homogeneous wetting according to Wenzel for PP.

In order to evaluate the influence of the wetting behavior on the joint strength, hybrid joints are fabricated using microstructured stainless steel and PA6/GF47 and PP/ GF40. For PA6/GF47, the joint strength strongly varies and high tensile shear strengths according to previous works [1] are not achieved. For PP/GF40, the joint strength for all parameters vary between 22 and $25 \mathrm{MPa}$ and show no direct correlation to the contact angle measurements. Also, the results show that CLP structures are not mandatory to enable a strong connection between the materials.

It can be concluded that there is no direct influence of the wetting properties of the metal surface onto the joint strength of the hybrid joint, although the surfaces show a strong hydrophobic behavior. Due to the applied joining pressure, the wettability is not significantly relevant for the joining process.

The presented results leave some open questions for further investigations regarding the causes for the reduced joint strength of PA6, the wettability of PP with smaller grain sizes/drop sizes, and the need to fully cover the surface with CLP structures for high joint strength.

Open Access This article is distributed under the terms of the Creative Commons Attribution 4.0 International License (http:// creativecommons.org/licenses/by/4.0/), which permits unrestricted use, distribution, and reproduction in any medium, provided you give appropriate credit to the original author(s) and the source, provide a link to the Creative Commons license, and indicate if changes were made.

\section{References}

1. van der Straeten K, Olowinsky A, Gillner A (2018) Laser-based plastic-metal-joining with self-organizing microstructures considering different load directions. J Laser Appl 30(3)

2. van der Straeten K, Burkhardt I, Olowinsky A, Gillner A (2016) Laser-induced self-organizing microstructures on steel for joining with polymers. Phys Procedia 83:1137-1144 
3. van der Straeten K, Engelmann C, Olowinsky A, Gillner A (2018) Comparison of laser-based joining approaches for plastic-metalhybrids - strength vs. process speed. Proceedings of Hybrid Materials and Structures, Bremen

4. Eifel S, Dohrn A, Gillner A Quality aspects in high power ultra-short pulse laser ablation. Proceedings of LPM2010, Stuttgart

5. Tsukamoto M, Kayahara T, Nakano H, Hashida M, Katto M, Fujita M, Tanaka M, Abe N (2007) Microstructures formation on titanium plate by femtosecond laser ablation. J Conf Ser 59:666-669 Eighth International Conference on Laser Ablation
6. Habenicht G (2009) Kleben - Grundlagen, Technologien, Anwendungen (German), 6th edn. Springer Verlag, Heidelberg

7. Wenzel RN (1936) Resistance of solid surfaces to wetting by water. Ind Eng Chem 28:988-994

8. Cassie ABD, Baxter S (1944) Wettability of porous surfaces. Trans Faraday Soc 40:546-551

Publisher's note Springer Nature remains neutral with regard to jurisdictional claims in published maps and institutional affiliations. 